\title{
Cognitive Effects of Lacosamide in Patients with Drug Refractory Focal Epilepsy: A Prospective Observational Study
}

\author{
Dr. Sophia B Modi * MD, Dr. Asha S, MD, Dr. Thomas Iype, MD, MRCP(UK), FRCP(Edin), DM, \\ Dr. Libu Gnanaseelan Kanakamma, MD, Mrs. Reeja Rajan
}

Government Medical College, Thiruvananthapuram, Kerala, India

*Corresponding Author: Dr Sophia B Modi; Assistant Professor, Department of Pharmacology, Government Medical College, Thiruvananthapuram, Kerala, India; sophiamodi@yahoo.com

Received 07 December 2021;

Accepted 22 December 2021;

Published 25 December 2021

\begin{abstract}
Objectives: This study was conducted to obtain data on the cognitive effects of lacosamide in Indian population. Methodology: An open labelled prospective observational study in 22 patients who suffered from focal epilepsy. Results: All the pre and post lacosamide cognition scores showed statistically significant positive correlation in this study. Average initial seizure frequency per month was 3.56 (SD 2.58 ) and median frequency 2.5 seizures per month. Range being 1-8 per month. At the final follow-up at 6 months, $87.5 \%$ of the study subjects had no seizures. In the remaining $12.5 \%$ of patients, reduction in seizure frequency was observed. The difference in frequency is statistically significant (Wilcoxon Signed Ranks Test P <0.001). Conclusion: Excellent seizure control is observed in patients with refractory focal epilepsy treated with lacosamide. Also, lacosamide has no serious adverse effects or drug interactions. In this study, it is observed that unlike many AEDs, lacosamide contributed to significant improvement in cognition.
\end{abstract}

Keywords: Lacosamide, Drug refractory epilepsy, Cognition, Adverse effects, New drug

\section{Introduction}

Epilepsy is one of the most common disorders of the brain affecting around 50 million people worldwide. ${ }^{[1]}$ Despite the advent of new antiepileptic drugs (AEDs) over the past 15 years, the treatment of uncontrolled partial-onset seizures remains a dilemma. The newer AEDs offer new mechanisms of action and better safety profiles than the previously used AEDs.

Lacosamide (LCM) is one of the third generation AEDs approved for adjunctive use in partial-onset seizures. It differs from other approved AEDs in that it has two novel mechanisms of action and favorable pharmacokinetic and safety profiles. Lacosamide is a chemical compound with anticonvulsant and antinociceptive properties. It was approved in 2008 by FDA for adjunctive therapy in refractory partial onset seizures in patients 17 years of age and older ${ }^{[2,3]}$. Lacosamide has a different mechanism of action compared to other AEDs. It has a dual mode of action. Lacosamide selectively enhances slow inactivation of voltagegated sodium channels, resulting in stabilization of hyper excitable physiological neuronal activity. It does not affect fast inactivation. Classical anticonvulsant drugs such as carbamazepine, phenytoin, and lamotrigine act on fast inactivation of voltage-dependent sodium channels. In addition, Lacosamide binds to the collapsin response mediator protein-2 (CRMP-2) a protein mainly expressed in the central nervous system and involved in neuronal differentiation and axonal outgrowth and modulates mCRMP-2 function in vitro. Modulation of CRMP-2 by lacosamide may contribute to the decreased neuronal loss observed in status epilepticus animal models and its potential antiepileptic effects as seen in animal models ${ }^{[4,5]}$.

More than $30 \%$ of epilepsy patients remain refractory to pharmacotherapy despite the advent of new antiepileptic drugs (AEDs) over last two decades ${ }^{[6]}$. A small percentage of these refractory patients may become seizure free when a new AED is added. Combined administration of AEDs or the application of novel AEDs is a therapeutic option especially when surgical treatment cannot be offered.

Patients with epilepsy frequently experience cognitive dysfunctions. A variety of factors contribute to their cognitive dysfunction. Because antiepileptic drugs (AEDs) are the major therapeutic modality for epilepsy, the adverse effects of AEDs on cognition are important. AEDs display a dose dependent effect on 
cognitive functioning, and even more striking adverse effects on cognition may result from AED polytherapy ${ }^{[7]}$. Some AEDs (e.g., topiramate) influence basic cognitive functions, such as alertness and attention, which secondarily may result in memory impairment.

On retrospective analysis of pooled safety data from 3 lacosamide double-blind phase II/III studies, ${ }^{[2,8-10]}$ the incidence of treatment emergent adverse events (TEAEs) related to cognition were dose dependent, being $1.9 \%$ for the lacosamide $200 \mathrm{mg} /$ day group (OR 0.4; 95 percent CI: 0.1-1.3), 8.5 percent for lacosamide $400 \mathrm{mg} /$ day (OR 1.7; 95 percent CI: $1.0-3.2$ ), and 13.8 percent at the unapproved $600 \mathrm{mg} / \mathrm{day}$ dose (OR 2.8; 95 percent CI 1.3-5.7). The TEAE related to cognition on administration of approved doses of lacosamide $(200 \mathrm{mg} / 400 \mathrm{mg})$ was 2.1 percent for lacosamide $400 \mathrm{mg} /$ day versus 0.4 percent for lacosamide 200 $\mathrm{mg} /$ day. Other spontaneously reported TEAEs included: memory impairment (1.5 percent for lacosamide $400 \mathrm{mg} /$ day\&, 1.1 percent for lacosamide $200 \mathrm{mg} /$ day), confusional state (1.5 percent for lacosamide $400 \mathrm{mg}$ /day \& 0.0 percent for lacosamide $200 \mathrm{mg}$ /day) and disturbance in attention (1.1 percent for lacosamide $400 \mathrm{mg}$ /day\& 0.0 percent for lacosamide $200 \mathrm{mg}$ /day).

In a retrospective, non-interventional, controlled longitudinal study ( $\mathrm{n}=44$ ), comparing lacosamide with lamotrigine and topiramate, lacosamide was observed to have only a minimal effect on cognition. $23 \%$ patients showed significant improvement in executive functions and $14 \%$ patients deteriorated in executive functions. Only one patient (1.6\%) showed significant deterioration in memory whereas 6 patients $(9 \%)$ showed significant improvement ${ }^{[11]}$.

There have been 3 phase II/III studies and one retrospective, non-interventional, controlled longitudinal study conducted in natural settings to assess oral lacosamide as adjunctive therapy ${ }^{[8,11-13]}$. These studies have been mainly conducted in the developed countries. Formal neuropsychological data are inadequate on cognitive effects of lacosamide especially in Indian population. The rationale behind conducting this study is to obtain data on the cognitive effects of lacosamide in Indian population.

\section{Objectives}

- $\quad$ To assess the effects of lacosamide on cognition among patients with drug refractory focal epilepsy to whom lacosamide is given as adjuvant therapy.

- To study the seizure control profile of lacosamide in focal epilepsy.

\section{Methodology}

This open labelled prospective observational study was done at Government Medical College, Thiruvananthapuram. Patients were recruited from epilepsy patients attending the Neurology OPD from January 2012 to December 2012. We recruited patients with drug refractory focal epilepsy who were given an adequate trial of at least two AEDs to maximum tolerated dose. Patients from both genders were included who had seizures of more than 6 months duration with a seizure frequency of at least 2 seizures in previous 3 months. We excluded patients who were pregnant, had renal or hepatic dysfunction, patients with any progressive, demyelinating, or degenerative neurological condition, with psychosis, alcohol\& substance abuse. We also excluded patients with active suicidal plan/intent or active suicidal thoughts in the last 6 months and those with prior history of cardiac arrhythmia.
Sample size and selection: According to the prescription rate of lacosamide at the study setting, we expected a sample size of 30 . Only 25 patients were prescribed lacosamide during the study period. Of them, 22 satisfied the inclusion criteria and they were studied.

\section{Study Tools}

- $\quad$ Pre-tested Proforma

- Malayalam adaptation of Addenbrooke's cognitive examination (M-ACE) to assess six cognitive domains, namely orientation, attention/concentration, memory, verbal fluency, language and visuospatial abilities to produce six sub-scores, contributing to the total score.

- Digit backward memory span to measure attention, concentration and mental control.

- Block design to measure spatial perception, visual abstract processing and problem solving.

- Digit symbol test to measure psychomotor speed and sustained attention.

- Trail making test- part A to examine cognitive processing speed.

- Engel's Seizure Scoring System

- Naranjo's score to record severity of adverse effects and their relationship to lacosamide

- Hospital Anxiety Depression Score

Ethics committee clearance was obtained, informed consent was obtained from each subject / guardian/ relative and confidentiality and anonymity of the patient's information were maintained.

\section{Data collection}

This study comprehended minimum 8 visits- an initial visit, follow up visits every 2 weeks in the initial 2months and monthly follow up visits for the next 4 months. Every subject was followed up at least for a period of 6 months after reaching the maintenance dose of lacosamide.

At the first visit, socio demographic and clinical details were collected. Baseline evaluation of the seizure type and frequency was done. Baseline cognition was assessed. Engel system was used to score seizure burden at the first visit. Seizure frequency and disability is scored in Engel system on a quasilogarithmic scale as a score ranging from 0-12. Scores less than 5 denote no seizures or non-disabling seizures (aura or brief partial seizures without impairment of consciousness or loss of muscular tone) only. Score 5 indicates 1 to 3 seizures per year; score 6 indicates 4-11 seizures per year. Seizure frequency of 1 per month and above scores $7-12^{[14]}$.

Following these baseline assessments, lacosamide was prescribed.

At the second to seventh OPD visit, assessment of vital signs and weight followed by physical and neurological examinations, seizure frequency, and adverse effects were done. Naranjo's scoring for recording severity of adverse effects and their relationship to lacosamide and Engels scoring were also done.

On the eighth OPD visit, apart from all other routine tests, battery of cognitive tests was repeated.

Collected data was entered in excel and analyzed using SPSS16. Relevant variables were expressed as means and standard deviation, medians, proportions and their $95 \% \mathrm{CI}$, appropriately. 
Appropriate statistical tests (Wilcoxon Signed Ranks Test, Mann-Whitney U, Kruskal-Wallis Test, One sample t test, Chisquare test, Fisher's exact, Spearman's correlation etc.) were performed to find out the statistical significance of changes in the cognitive variables and association with other variables studied.

\section{"Standard Protocol Approvals, Registrations and Patient Consents"}

a) I received approval from an ethical standards committee on human experimentation (institutional or regional) for any experiments using human subjects

b) Written informed consent was obtained from all patients (or guardians of patients) participating in the study (consent for research).

\section{Results}

Average age of the study population was 36.05 . Majority were males $(63.6 \%)$. $59.1 \%$ of the subjects were socially active. $31.8 \%$ had high school education and $27.3 \%$ had higher education. Most of the study subjects belonged to Below Poverty Line category $(81.8 \%)$. Family history of seizure disorder was reported by 2 (9\%) subjects.

\section{Baseline characteristics}

In the present study, the most common time of onset of epilepsy was adolescence (31.8\%) the next being childhood (27.3\%) and mean duration of epilepsy in the subjects was 20.8 years. Average baseline seizure frequency per six months was 21.4. Aura was present only in $23 \%$ of patients. $27.3 \%$ subjects had EEG abnormalities and $45.5 \%$ had CT/MRI abnormalities. Epileptiform abnormalities in EEG had temporal localization in 4 subjects and multifocal epileptiform abnormalities were seen in 2 subjects. The MRI / CT abnormalities included chronic infarcts; focal atrophy of right frontal lobe, mild atrophic changes of brain parenchyma and cerebellum; mild diffuse atrophy of right hippocampus, amygdala, right column of fornix, right mammillary body; right hemisphere atrophy; gliotic changes in right posterior temporal lobe with gross parenchymal atrophy; volume loss of left hippocampus, mesial sclerosis; bilateral lateral \&third ventricle enlargement, hyperintense signal densities in bilateral cerebral hemisphere and periaqueductal region; bilateral frontal heteropia with polymicrogyria etc.

Most of the patients in this study were receiving 3 AEDs (59.1\%) concomitantly. Most common AEDs the subjects were receiving were carbamazepine $(68.2 \%)$ followed by valproate (22.7\%). 72.7\% were getting sodium channel blocking AEDs like carbamazepine, phenytoin and topiramate concomitantly. In this study, $16(72.7 \%)$ subjects continued lacosamide till final follow up. Lacosamide was discontinued by $27.2 \%$ patients; of whom $22.7 \%$ discontinued the drug themselves giving reasons such as dizziness $(13.5 \%)$ and financial burden (9\%). Mean dose of lacosamide was $296.88 \mathrm{mg} /$ day. (Median dose 300mg/day)

Among the 22 patients, 10 patients $(45 \%)$ complained of dizziness. Dizziness was reported mostly during titration than during the maintenance phase. Withdrawal of patients from this study due to dizziness was more during the titration phase. 12 patients (55\%) tolerated lacosamide well without any adverse drug reactions. Of the 16 followed up till 6 months, 7(43.8\%) complained about dizziness. Though not significant, those who were concomitantly receiving other sodium channel blocking AEDs had higher frequency of dizziness compared to subjects receiving non-sodium channel blocking AEDs. Dizziness showed no association with type of epilepsy, CT/MRI abnormalities or EEG abnormalities. Comparison of on-treatment versus baseline ECG results did not show any change in heart rate, PR interval, QT interval, or QRS duration in the study subjects.

\section{Baseline Cognition}

In the baseline evaluation, majority of the patients showed cognitive impairment. Difference in mean M-ACE total score from population normal [88] was statistically significant. No significant association was observed between baseline M-ACE scores with most of the socio demographic and clinical variables studied. Orientation showed a positive correlation with years of schooling $(\mathrm{P}<0.05)$.

Of the 22 subjects, only $72.7 \%$ were able to perform Trail making test- part A. Mean baseline time taken to complete the test was 78.4 seconds ${ }^{[38]}$. Difference in mean time taken by subjects from population average (29 seconds) was statistically significant $(\mathrm{P}<0.05)$. Mean score of digit span backward was 2.1, which was less than normal ( $4 \pm 1$ depending on age and intellectual abilities).

No significant association was observed between baseline TMT-A, digit backward, digit symbol and block design test scores with most of the socio demographic and clinical variables except TMT-A with education status (P 0.018) and Block design with Occupation. (P 0.021)

In this study, it was observed that, base line Trail making test Part-A (TMT-A), digit backward and digit symbol tests are correlated with years of schooling $(\mathrm{P}<0.05)$.

\section{Pre and Post Lacosamide Cognition}

Difference in pre and post lacosamide M-ACE total scores was not significant ( $\mathrm{p}$ 0.139). (Figure 2) Difference in Memory showed statistically significant improvement ( $\mathrm{P}$ 0.007). Naming showed statistically significant improvement (P 0.037). 


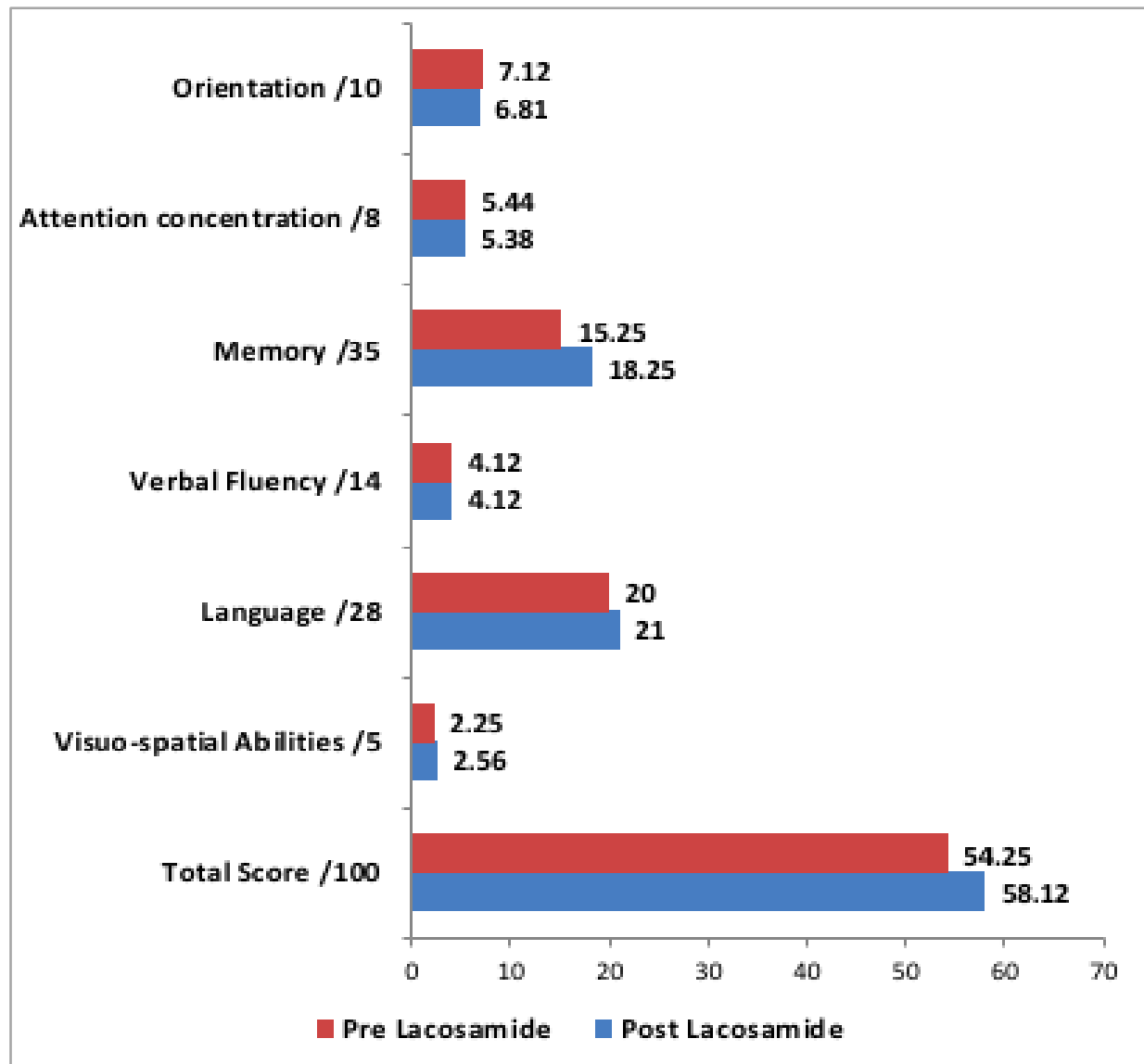

Figure 1: Clustered bar diagram comparing pre lacosamide and post lacosamide M-ACE scores

In this study, only $31.25 \%$ subjects showed a reduction in M-ACE total score from the baseline value. (Table1) Maximum number of people showed deterioration in sub-domain orientation $37.5 \%$ and minimum in memory and visuo-spatial abilities $18.75 \%$ each. (Figure3) The association between number of AEDs and attention/concentration was statistically significant ( $\mathrm{p}$ 0.03). Subjects from higher socio-economic status were having better improvement in total cognition scores (P 0.03) and sub-domain language (P 0.03) compared to subjects from low socio-economic background.

Table 1: Pre lacosamide and post lacosamide M-ACE scores and their significance

\begin{tabular}{|l|l|l|l|l|l|l|l|l|}
\hline & \multicolumn{2}{|l|}{ Pre Lacosamide } & \multicolumn{3}{l|}{ Post Lacosamide } & \multicolumn{3}{l|}{ Pre and Post Lacosamide Difference } \\
\hline & Mean & Median & Mean & Median & \multicolumn{2}{l|}{ Mean (SD) 95\%CI } & Sig \\
\hline Orientation & 7.12 & 8.5 & 6.81 & 7.5 & $-0.31(1.92)$ & -1.25 & 0.63 & P 0.5 \\
\hline Attention/ Concentration & 5.44 & 6 & 5.38 & 6 & $-0.06(2.14)$ & -1.11 & 0.99 & P 0.9 \\
\hline Memory & 15.25 & 15.5 & 18.25 & 19.5 & $3(3.86)$ & 1.11 & 4.89 & P 0.007 \\
\hline Verbal fluency & 4.12 & 4 & 4.12 & 4 & $0(3.16)$ & -1.55 & 1.55 & P 0.89 \\
\hline Language & 20 & 21 & 21 & 23.5 & $0.94(3.21)$ & -0.64 & 2.51 & P 0.053 \\
\hline Visuo-spatial Abilities & 2.25 & 2 & 2.56 & 3.5 & $0.31(1.01)$ & -0.18 & 0.80 & P 0.19 \\
\hline Total Score & 54.3 & 60.5 & 58.1 & 63 & $3.88(10.7)$ & -1.37 & 9.12 & P 0.13 \\
\hline
\end{tabular}

*Wilcoxon Signed Ranks Test

Table 2: Correlation between differences in pre and post lacosamide cognition scores (M-ACE, Trail making test-part A, digit backward test, digit symbol test, block design test)

\begin{tabular}{|l|l|l|}
\hline Pre Vs. Post lacosamide scores & Spearman's rho & P value \\
\hline Orientation & 0.774 & $<0.001$ \\
\hline Attention Concentration & 0.506 & 0.045 \\
\hline Memory & 0.603 & 0.013 \\
\hline Verbal Fluency & 0.19 & 0.47 \\
\hline Language & 0.85 & $<0.001$ \\
\hline Visuospatial abilities & 0.88 & $<0.001$ \\
\hline Total score (M-ACE) & 0.78 & $<0.001$ \\
\hline Trail making test part-A & 0.666 & 0.025 \\
\hline Digit backward & 0.822 & $<0.001$ \\
\hline Digit symbol & 0.889 & $<0.001$ \\
\hline Block design & 0.496 & 0.051 \\
\hline
\end{tabular}




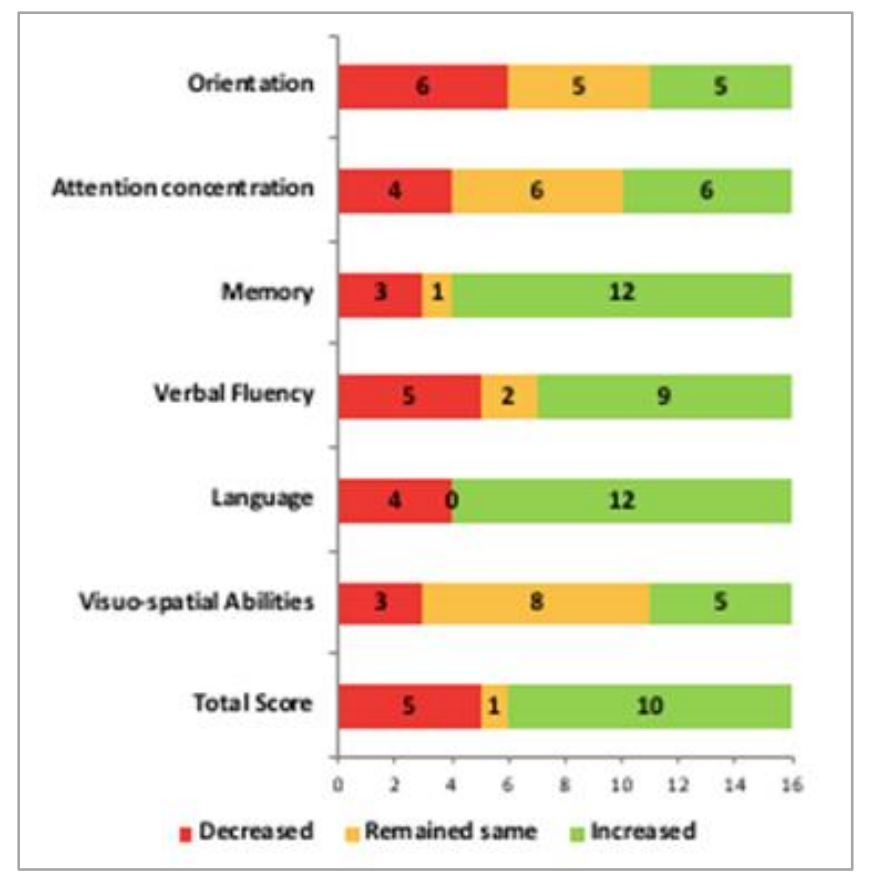

Figure 2: Stacked bar showing proportion of subjects who showed changes in cognition during follow up according to $M$ ACE scores.

It was observed that increase in number of AEDs and dose of lacosamide decreases cognition scores. Negative correlation was shown by attention /concentration, memory, verbal fluency and total score with number of AEDs. Difference in pre and post lacosamide language score showed statistically significant positive correlation with years of schooling. Subjects getting four AEDs showed deterioration in post lacosamide M-ACE total scores compared to those who got three or two AEDS.

Post lacosamide digit backward test scores (which assess attention and working memory) showed significant improvement from baseline scores (P 0.04). Digit symbol (which measures psychomotor speed and sustained attention) and block design tests (which measures spatial perception, visual abstract processing, and problem solving) showed improvement, but not significant. In this study, $25 \%$ of subjects showed post lacosamide deterioration in TMT-A performance and only one subject showed deterioration in digit backward test.

Difference in TMT-A showed association with marital status (P 0.042) and educational status of the subjects (P 0.019). Digit backward test also showed association with occupation status of the subjects (P 0.047). It shows that subjects with higher education took lesser time for completing TMT-A in post lacosamide situation.

Significant association was observed between CT/MRI abnormalities Vs. TMT-A (P 0.016). EEG abnormalities showed significant association with Digit Symbol (P 0.03) and Block design (P 0.042) tests.

All the pre and post lacosamide cognition scores except verbal fluency and block design showed statistically significant positive correlation in this study. (Table 2)

It was observed in this study that as age advances, time taken to complete TMT-A increases, which is an adverse cognitive effect.

\section{Seizure Control Profile}

$87.5 \%$ of the study subjects had no seizures at 6 months of follow up. In the remaining $12.5 \%$ of patients, reduction in seizure frequency was observed. The difference in seizure frequency before and after lacosamide administration is statistically significant $(\mathrm{P}<0.001)$. Engel's seizure score showed rapid decline following introduction of lacosamide. After 3rd month it became low and remained steady, showing excellent seizure control. (Figure 1) The difference between initial and final Engel's score is statistically significant $(\mathrm{P}<0.001)$.

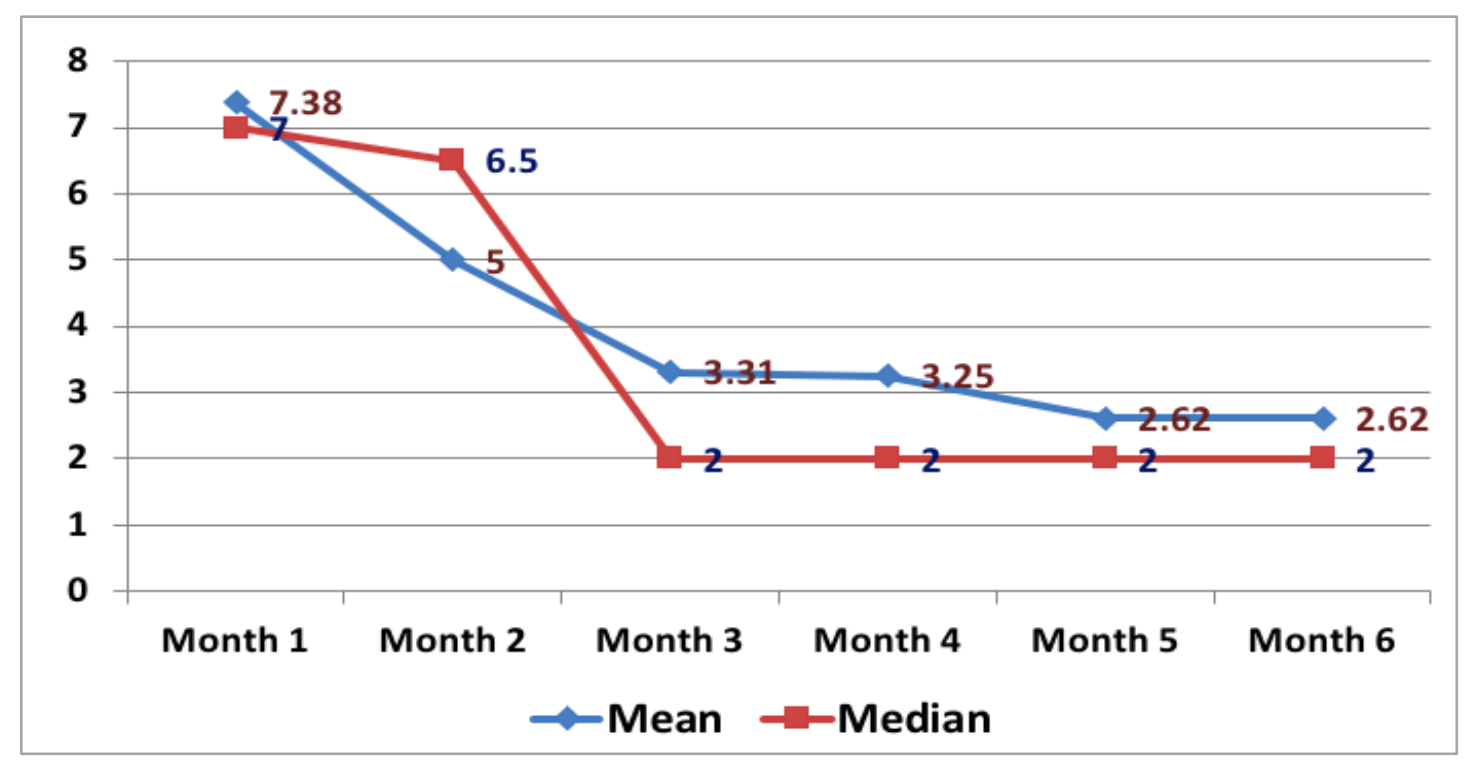

Figure 3: Engel's Seizure score during the follow up period.

\section{Discussion}

Cognitive impairment is a common secondary consequence of epilepsy. Several interacting factors including age of onset, seizure (type, duration, frequency, severity \& etiology), hereditary factors, psychosocial issues and cognitive side effects of antiepileptic drug (AED) therapy contribute to the cognitive dysfunctions ${ }^{[15]}$. Anti- epileptic drugs control seizures by causing global changes in the excitation levels in central nervous system. This alteration in neuronal excitation levels may result in cognitive deficits. Cognitive dysfunctions can negatively affect tolerability, compliance, and long-term retention of the treatment and can significantly affect every day functioning and quality of life of the patient. Lacosamide is a newer AED approved in 2008 for the 
treatment of refractory focal epilepsy. Very few studies have systematically examined the cognitive side effects of lacosamide. This study was aimed at assessing the effect of lacosamide on cognitive functions in patients with focal epilepsy attending the neurology department of a tertiary care hospital.

Similar to discontinuation rate in this study, Nunes et al also observed that discontinuation rate has been seen to be higher in lacosamide treated patients when compared to other AEDs ${ }^{[15]}$. In a similar study by Flores et al, 38\% patients discontinued lacosamide, of which $20.8 \%$ withdrew due to intolerable ADRs alone ${ }^{[17]}$.

Similar to the findings in this study where $45 \%$ complained of dizziness, Flores et al observed that $48.7 \%$ patients reported adverse effects. Sedation and dizziness were the most common ADRs followed by nausea ${ }^{[17]}$. The incidence of dizziness due to lacosamide in various studies range from $10.4 \%$ to $44.5 \%{ }^{[18,19,13,2]}$. It was observed that median dose of Lacosamide was equal (300mg/day) among both groups. Adverse events were not seen to be dose dependent in this study. But in many studies, adverse events were observed to be dose dependent ${ }^{[20]}$.

Though not significant, those who were concomitantly receiving sodium channel blocking AEDs in this study had higher frequency of dizziness compared to subjects receiving non-sodium channel blocking AEDs. This is in accordance with the study by Novy et al, in which lacosamide when concomitantly administered with other sodium channel blocking AEDs was observed to cause increased side effects (diplopia, dizziness, drowsiness) ${ }^{[21]}$.

In the present study, comparison of on-treatment versus baseline ECG results did not demonstrate any change in heart rate, PR interval, QT interval, or QRS duration in the study subjects. No cardiac adverse events were reported during the study period. Cardiac adverse effects including dose dependent PR interval prolongation, first and second-degree $\mathrm{AV}$ block and atrial fibrillation/flutter has been observed with lacosamide in few studies ${ }^{[22-24]}$. None of the subjects in the current study had any cardiac conduction problems or were taking drugs known to cause PR interval prolongation or had severe cardiac disease such as myocardial ischemia or heart failure at base line. This factor might be the reason for the lack of cardiac adverse events observed in the present study.

Similar to the $13.5 \%$ participant withdrawal from this study due to dizziness experienced during treatment with lacosamide, percentages of withdrawals during the lacosamide treatment period because of adverse effects were $8 \%, 8.7 \%, 17 \%{ }^{[2,13,25]}$ etc. with dizziness, ataxia, nausea, vomiting, diplopia etc. being the most common reasons in various studies ${ }^{[26]}$. Causality of ADR assessed by Naranjo's Algorithm showed $100 \%$ probable reactions.

It was observed in this study that verbal fluency remained same in pre and post lacosamide assessment. Language, visuospatial abilities and total score showed improvement. Difference in Memory showed statistically significant improvement (P 0.007). Recall and Retrograde memory showed statistically significant improvement. (Recall P 0.02). Retrograde Memory (P 0.021)). Naming showed statistically significant improvement (P 0.037).

In a similar study, Helmstaedter observed that $9 \%$ of patients treated with lacosamide showed significant improvement in memory functions ${ }^{[11]}$. A meta-analysis of 10 lacosamide randomized controlled trials for various indications observed that lacosamide is not associated with any significant cognitive adverse events. Memory impairment even though reported was found to be statistically not significant ${ }^{[27]}$.
The association between number of AEDs and attention/concentration was statistically significant (P 0.03). The observation in the present study is similar to the observation by Meador KJ that polytherapy (co-administration of multiple anticonvulsants) contributes to the risk of cognitive dysfunction and also increases the intensity of cognitive dysfunction ${ }^{[28]}$.

In this study, significant correlation was observed between pre and post lacosamide M-ACE total scores and all sub domain scores except Verbal fluency. (Table 2) Negative correlation was shown by attention /concentration, memory, verbal fluency and total score with number of AEDs. But years of schooling showed a positive correlation with M-ACE total score and most of its subdomains. This goes in accordance with Mathuranath et al who observed that level of education is the demographic factor that significantly affects the M-ACE total score in Malayalam speaking population in southern India ${ }^{[29,30]}$.

Post lacosamide digit backward test scores (which assess attention and working memory) showed significant improvement from baseline scores (P 0.04). In this study, $25 \%$ of subjects showed post lacosamide deterioration in TMT-A performance and only one subject showed deterioration in digit backward test. This goes in accordance with Helmstaedter et al. who observed that $23 \%$ of patients treated with lacosamide showed significant improvement in executive functions and $14 \%$ patients deteriorated in executive functions ${ }^{[11]}$.

All the pre and post lacosamide cognition scores showed statistically significant positive correlation in this study. (Table 3) Of the subjects with EEG abnormalities, 50\% showed deterioration in psychomotor speed and sustained attention from baseline while none with normal EEG deteriorated. Statistically significant ( $P$ $0.05)$.

Difference in TMT-A showed association with marital status (P 0.042) and educational status of the subjects (P 0.019). Digit backward test also showed association with occupation status of the subjects (P 0.047). It shows that subjects with higher education took lesser time for completing TMT-A in post lacosamide situation.

Significant association was observed between CT/MRI abnormalities Vs. TMT-A (P 0.016). EEG abnormalities showed significant association with Digit Symbol (P 0.03) and Block design (P 0.042) tests. This goes in accordance with Hermann et.al. who observed that baseline volumetric abnormalities are predictive of an increased risk of a progressively abnormal cognitive course [31].

In this study, while looking at correlation, all tests except digit symbol showed a positive correlation. It was observed in this study that as age advances, time taken to complete TMT-A increases, which is an adverse cognitive effect. This goes in accordance with Tombaugh et al and Ashendorf et al who have observed that TMT- A performance declines with increasing age but not with education ${ }^{[32,33]}$.

$87.5 \%$ of the study subjects had no seizures at 6 months of follow up. In the remaining $12.5 \%$ of patients, reduction in seizure frequency was observed. In patients who had secondary generalized tonic-clonic seizures (GTCS), the seizure type changed to complex partial seizures (CPS). If a patient who used to experience GTCS earlier is experiencing only simple partial seizures and CPSs later on, then it might be a sign of improved control of the seizures. In the present study, all patients reported improvement in seizure severity.

The difference in seizure frequency prior to lacosamide administration and post lacosamide is statistically significant $(\mathrm{P}$ 
$<0.001)$. This goes in accordance with various similar studies, where the median percentage reduction in seizure frequency per 28 days from baseline to the maintenance period for lacosamide ranged from $32.7 \%$ to $35.3 \%$ for $200 \mathrm{mg} / \mathrm{day}$ and $36.4 \%$ to $41.1 \%$ for $400 \mathrm{mg} /$ day ${ }^{[2,8,13]}$.

Engel's seizure score rapidly declined following introduction of lacosamide. It became low and remained steady after the 3rd month, showing excellent seizure control. The difference between initial and final Engel's score is statistically significant $(\mathrm{p}<0.001)$.

Multiple factors might have played a role in the improvement in cognitive functions observed in this study. One factor can be the significant reduction in seizure frequency. It is a proved fact that continuing seizures contribute to cognitive deterioration in epileptic patients by inducing hippocampal sclerosis ${ }^{[34]}$. The reduction in seizure severity might also be an important contributor to the cognitive improvement. Previous studies have shown that severe seizures and status epilepticus reduces cognition ${ }^{[35-37]}$. Another important factor might be the reduction in dose of or stopping of other concomitant AEDs which are associated with cognitive deterioration(phenobarbitone, carbamazepine etc.).

\section{Limitations}

This study was conducted as a prospective observational study. For better assessment of association, an analytical study, preferably a randomized controlled trial is desirable. Another important limitation was the small number of subjects included in this study. This was because lacosamide is a comparatively new drug and so it is being prescribed only to patients in whom treatment with first line AEDs fail. Another limitation was the limited period of monitoring of adverse drug reactions. For complete assessment of adverse effects of lacosamide on cognition, patients have to be followed up over a longer time.

\section{Conclusion}

In the above study, it is observed that unlike many AEDs, lacosamide contributed to significant improvement in cognition. Excellent seizure control is observed in patients with refractory focal epilepsy treated with lacosamide. Also, lacosamide has no serious adverse effects or drug interactions.

\section{Summary box}

1. Lacosamide contributed to significant improvement in cognitive functions in patients with refractory focal epilepsy.

2. Significant reduction in seizure frequency and seizure severity is observed in patients with refractory focal epilepsy treated with lacosamide.

3. Lacosamide has no serious adverse effects or drug interactions.

\section{Declarations}

\section{Ethics approval}

Ethics committee clearance was obtained, informed consent was obtained from each subject / guardian/ relative and confidentiality and anonymity of the patient's information were maintained.

\section{Consent to participate}

Informed consent was obtained from each subject / guardian/ relative

\section{Consent for publication}

Informed consent was obtained from each subject / guardian/ relative

\section{Funding}

Nil

\section{Conflicts of interest/Competing interests}

Nil

\section{Authors' contributions}

Study conception, design and material preparation: Dr Sophia B Modi, Dr Thomas Iype and Dr Asha S

Data collection: Dr Sophia B Modi, Dr Thomas Iype and Mrs Reeja Rajan.

Formal data analysis: Dr. Libu Gnanaseelan Kanakamma

Writing - original draft: Dr Sophia B Modi

Writing - review \& editing: Dr Asha S, Dr Sophia B Modi, Dr Thomas Iype

All authors read and approved the final manuscript.

\section{References}

[1] Epilepsy [Internet]. [cited 2021 Mar 9]. Available from: https://www.who.int/news-room/fact-

sheets/detail/epilepsy

[2] Chung S, Ben-Menachem E, Sperling MR, Rosenfeld W, Fountain NB, Benbadis S, et al. Examining the clinical utility of lacosamide: pooled analyses of three phase II/III clinical trials. CNS Drugs. 2010 Dec;24(12):104154.

[3] Vimpat ${ }^{\circledR}$ - Epilepsy | UCB [Internet]. [cited 2021 Mar 9]. Available from: https://www.ucb.com/ourproducts/Products/vimpat\%C2\%AE-epilepsy

[4] Lacosamide: a review of preclinical properties - PubMed [Internet]. [cited 2021 Mar 9]. Available from: https://pubmed.ncbi.nlm.nih.gov/17461888/

[5] Effects of the Novel Antiepileptic Drug Lacosamide on the Development of Amygdala Kindling in Rats - Brandt - 2006 - Epilepsia - Wiley Online Library [Internet]. [cited 2021 Mar 9]. Available from: https://onlinelibrary.wiley.com/doi/full/10.1111/j.15281167.2006.00818.x

[6] Granata T, Marchi N, Carlton E, Ghosh C, GonzalezMartinez J, Alexopoulos AV, et al. Management of the patient with medically refractory epilepsy. Expert Rev Neurother. 2009 Dec;9(12):1791-802.

[7] Kim S-Y, Lee H-W, Jung D-K, Suh C-K, Park S-P. Cognitive Effects of Low-dose Topiramate Compared with Oxcarbazepine in Epilepsy Patients. J Clin Neurol. 2006 Jun;2(2):126-33.

[8] Ben-Menachem E, Biton V, Jatuzis D, Abou-Khalil B, Doty P, Rudd GD. Efficacy and safety of oral lacosamide 
as adjunctive therapy in adults with partial-onset seizures. Epilepsia. 2007 Jul;48(7):1308-17.

[9] Lacosamide: efficacy and safety as oral adjunctive treatment for partial-onset seizures | Cochrane Library [Internet]. [cited 2021 Mar 9]. Available from: https://www.cochranelibrary.com/central/doi/10.1002/ce ntral/CN-00744961/related-content

[10] Adjunctive lacosamide for partial-onset seizures: Efficacy and safety results from a randomized controlled trial - PubMed [Internet]. [cited 2021 Mar 9]. Available from: https://pubmed.ncbi.nlm.nih.gov/19183227/

[11] Helmstaedter C, Witt J-A. The longer-term cognitive effects of adjunctive antiepileptic treatment with lacosamide in comparison with lamotrigine and topiramate in a naturalistic outpatient setting. Epilepsy Behav. 2013 Feb;26(2):182-7.

[12] Lacosamide: efficacy and safety as oral adjunctive treatment for partial-onset seizures | Cochrane Library [Internet]. [cited 2021 Mar 9]. Available from: https://www.cochranelibrary.com/central/doi/10.1002/ce ntral/CN-00744961/related-content

[13] Halász P, Kälviäinen R, Mazurkiewicz-Beldzińska M, Rosenow F, Doty P, Hebert D, et al. Adjunctive lacosamide for partial-onset seizures: Efficacy and safety results from a randomized controlled trial. Epilepsia. 2009 Mar;50(3):443-53.

[14] Engel J Jr, Ness V, Rasmussen, Ojemann LM. Outcome with respect to epileptic seizures. In: Surgical treatment of the epilepsies (Ed Engel J Jr). New York: Raven Press; 1993. p. 609-22.

[15] Epilepsy and Cognition - Bruce Hermann, Michael Seidenberg, 2007 [Internet]. [cited 2021 Mar 9]. Available from: https://journals.sagepub.com/doi/10.1111/j.15357511.2007.00151.x

[16] Nunes VD, Sawyer L, Neilson J, Sarri G, Cross JH. Profile of lacosamide and its role in the long-term treatment of epilepsy: a perspective from the updated NICE guideline. Neuropsychiatr Dis Treat. 2013;9:46776.

[17] Flores L, Kemp S, Colbeck K, Moran N, Quirk J, Ramkolea $\mathrm{P}$, et al. Clinical experience with oral lacosamide as adjunctive therapy in adult patients with uncontrolled epilepsy: a multicentre study in epilepsy clinics in the United Kingdom (UK). Seizure. 2012 Sep;21(7):512-7.

[18] Husain A, Chung S, Faught E, Isojarvi J, McShea C, Doty P. Long-term safety and efficacy in patients with uncontrolled partial-onset seizures treated with adjunctive lacosamide: results from a Phase III openlabel extension trial. Epilepsia. 2012 Mar;53(3):521-8.

[19] Side Effects of Drugs Annual: A worldwide yearly survey of new data in adverse drug reactions. Elsevier; $2015.693 \mathrm{p}$.

[20] Development of lacosamide for the treatment of partialonset seizures - PubMed [Internet]. [cited 2021 Mar 9]. Available from: https://pubmed.ncbi.nlm.nih.gov/23859801/

[21] Baulac M, Byrnes W, Williams P, Borghs S, Webster E, De Backer $\mathrm{M}$, et al. Lacosamide and sodium channelblocking antiepileptic drug cross-titration against levetiracetam background therapy. Acta Neurol Scand. 2017 Apr;135(4):434-41.
[22] Rauck RL, Shaibani A, Biton V, Simpson J, Koch B. Lacosamide in painful diabetic peripheral neuropathy: a phase 2 double-blind placebo-controlled study. Clin J Pain. 2007 Feb;23(2):150-8.

[23] Efficacy and safety of lacosamide in diabetic neuropathic pain: an 18-week double-blind placebo-controlled trial of fixed-dose regimens - PubMed [Internet]. [cited 2021 Mar 9]. Available from: https://pubmed.ncbi.nlm.nih.gov/19454870/

[24] Degiorgio CM. Atrial flutter/atrial fibrillation associated with lacosamide for partial seizures. Epilepsy Behav. $2010 \mathrm{Jul} ; 18(3): 322-4$.

[25] Chung SS, Kelly K, Schusse C. New and Emerging Treatments for Epilepsy: Review of Clinical Studies of Lacosamide, Eslicarbazepine Acetate, Ezogabine, Rufinamide, Perampanel, and Electrical Stimulation Therapy. J Epilepsy Res. 2011 Dec 30;1(2):35-46.

[26] Doty P, Hebert D, Mathy F-X, Byrnes W, Zackheim J, Simontacchi K. Development of lacosamide for the treatment of partial-onset seizures. Ann N Y Acad Sci. 2013 Jul;1291:56-68.

[27] Zaccara G, Perucca P, Loiacono G, Giovannelli F, Verrotti A. The adverse event profile of lacosamide: a systematic review and meta-analysis of randomized controlled trials. Epilepsia. 2013 Jan;54(1):66-74.

[28] Meador KJ, Loring DW. Developmental effects of antiepileptic drugs and the need for improved regulations. Neurology. 2016 Jan 19;86(3):297-306.

[29] Mathuranath PS, Cherian JP, Mathew R, George A, Alexander A, Sarma SP. Mini Mental State Examination and the Addenbrooke's Cognitive Examination: Effect of education and norms for a multicultural population. Neurology India. 2007 Apr 1;55(2):106.

[30] Mathuranath PS, Hodges JR, Mathew R, Cherian PJ, George A, Bak TH. Adaptation of the ACE for a Malayalam speaking population in southern India. Int $\mathrm{J}$ Geriatr Psychiatry. 2004 Dec;19(12):1188-94.

[31] Hermann B, Meador KJ, Gaillard WD, Cramer JA. Cognition across the lifespan: Antiepileptic drugs, epilepsy, or both? Epilepsy \& Behavior. 2010 Jan;17(1):1-5.

[32] Tombaugh TN. Trail Making Test A and B: normative data stratified by age and education. Arch Clin Neuropsychol. 2004 Mar;19(2):203-14.

[33] Trail Making Test errors in normal aging, mild cognitive impairment, and dementia - PubMed [Internet]. [cited 2021 Mar 10]. Available from: https://pubmed.ncbi.nlm.nih.gov/18178372/

[34] Trimble MR. Cognitive Hazards of Seizure Disorders. Epilepsia. 1988;29(s1):S19-24.

[35] Mula M, Trimble MR. Antiepileptic drug-induced cognitive adverse effects: potential mechanisms and contributing factors. CNS Drugs. 2009;23(2):121-37.

[36] Thompson PJ, Duncan JS. Cognitive decline in severe intractable epilepsy. Epilepsia. 2005 Nov;46(11):1780-7.

[37] Dodrill CB. Progressive cognitive decline in adolescents and adults with epilepsy. Prog Brain Res. 2002;135:399407.

[38] Reitan RM. Validity of the Trail Making Test as an Indicator of Organic Brain Damage. Perceptual and Motor Skills. 1958;8(3):271-6. 
c) (†)

Open Access This article is licensed under a

Creative Commons Attribution 4.0 International License, which permits use, sharing, adaptation, distribution and reproduction in any medium or format, as long as you give appropriate credit to the original author(s) and the source, provide a link to the Creative Commons license, and indicate if changes were made. The images or other third party material in this article are included in the article's Creative Commons license, unless indicated otherwise in a credit line to the material. If material is not included in the article's Creative Commons license and your intended use is not permitted by statutory regulation or exceeds the permitted use, you will need to obtain permission directly from the copyright holder. To view a copy of this license, visit https://creativecommons.org/licenses/by/4.0/.

(C) The Author(s) 2021 